\title{
PROCEEDINGS REPORTS
}

\section{Developing a clinician-scientist career}

\author{
Isabella T. Tai MD, PhD
}

Division of Gastroenterology, University of British Columbia and Genome Sciences Centre, BC Cancer Agency, Vancouver, Canada

Presented at the CSCI Symposium Clinician Investigators: Pathway to Future Success Winnipeg, September 2007

Clin Invest Med 2008; 31 (5): E300-E301.
It was a pleasure and an honour to be invited to participate in this year's CSCI symposium "Clinician Investigators: Pathway to Future Success". Looking out into the audience, it was satisfying to see the room filled with so many trainees representing the various $\mathrm{MD} / \mathrm{PhD}$ and CIP programs across Canada. As the first female graduate of University of Toronto's MD/ $\mathrm{PhD}$ program, it was also very encouraging to notice the large numbers of female trainees attending this conference. The following is a short synopsis of some key elements to consider as one transitions into a junior faculty position.

\section{Choosing a Clinician-Scientist Career}

Many of us begin to contemplate a career as a Clinician-Scientist because of the unique opportunity it provides: the prospect of conducting innovative research while providing clinical care to our patients. Traditionally, training for this "hybrid" career begins during medical school, by enrolling in $\mathrm{MD} / \mathrm{PhD}$ programs; or through the Clinician Investigator Program during post-graduate residency training. However, some individuals will enter this career path without formal enrolment in these established training programs, but by pursuing medical training and graduate studies as separate streams.
Ultimately, we choose to pursue the path of a Clinician Scientist because we share a passion for research and the hope that our clinical training will also bring a clearer focus to disease-oriented problems and solutions that will translate seamlessly from bench back to the bedside.

\section{Challenges Encountered as a Junior Faculty:}

How to decide on your first faculty position?

After the years of training (medical school, residency, post-doctoral research fellowship), the time has come to take on a "real job" and where to begin your Clinician-Scientist career is the most important next step. How does one decide which institution to join? One of the most important considerations is based on leadership and environment of the division (and department) one is considering at that institution. Ideally, both should have a track record of successfully nurturing and supporting their junior ClinicianScientists. As a wet-bench scientist, one should also consider the research environment, which is equally important: will the laboratory be located in close proximity to potential collaborators? Are resources available (core equipment, facilities) that will help develop your research program? These are important elements 
to consider as you visit the different institutions during your search for a faculty position.

\section{How to become a competitive researcher and a good clinician at the same time?}

Most of us are aware of the 75:25 (research:nonresearch) split "requirement" to succeed as a ClinicianScientist, but it is often not appreciated until most of us take on our first faculty position. This time commitment of at least $75 \%$ to be devoted to research is crucial for a junior Clinician Scientist (and as well, for more established investigators) in order to achieve success as a wet-bench researcher. In the first few years of entering a new faculty position, this "protected" time allows one to establish a research program (hiring new laboratory personnel, purchasing new equipments, while conducting experiments originating from one's own lab!). However, at the same time, in order to maintain good clinical skills, it becomes necessary to focus one's clinical practice by choosing a specialty.

\section{Salary and Grant support}

Salary and grant supports are both important. However, as a junior faculty, salary support is critical to ensuring adequate time protection for research. This support may be provided by internal institutional or departmental funds initially. At some institutions, there are competitive internal awards, however, the mainstay are the external competitive salary awards that are available either at the provincial or national levels. As for competing for grants to support one's research, there is a small window of opportunity provided by start-up funds before it becomes necessary to apply for competitive research grants. During this time, use start-up funds to support research projects (and some laboratory personnel) to generate preliminary data, which can form the basis of some initial grant applications.

\section{Mentorship}

Our mentors guided us during our training, and continue to do so as we move into our first faculty positions and beyond. Their continued guidance is vital as junior faculties wade through the "dos" and "don'ts" of establishing themselves as Clinician Scientists. The importance of these individuals and the role they play in contributing to the success of a faculty member at any level cannot be over emphasized.

Although the above may seem overwhelming, the transition from trainee to junior faculty is an incredibly exciting period, as one is finally given the opportunity to undertake the hybrid role that we have all been trained to pursue. Enjoy the journey to becoming a Clinician-Scientist career, as it is well worth pursuing!

Correspondence to:

Please change to:

Isabella T. Tai, MD, PhD

Assistant Professor

Division of Gastroenterology,

University of British Columbia and

Senior Scientist

Genome Sciences Centre,

BC Cancer Agency

itai@bcgsc.ca 